\title{
Role of phase partitioning in coordinating DNA damage response: focus on the Apurinic Apyrimidinic Endonuclease 1 interactome
}

https://doi.org/10.1515/bmc-2020-0019

received October 30, 2020; accepted November 16, 2020.

\begin{abstract}
Liquid-liquid phase separation (LLPS) is a way to concentrate biochemical reactions while excluding noninteracting components. Disordered domains of proteins, as well as interaction with RNA, favor condensation but are not mandatory for modulating this process. Recent insights about phase-separation mechanisms pointed to new fascinating models that could explain how cells could cope with DNA damage responses, conferring both spatial and temporal fine regulation. APE1 is a multifunctional protein belonging to the Base Excision Repair (BER) pathway, bearing additional 'non-canonical' DNA-repair functions associated with processes like RNA metabolism. Recently, it has been highlighted that several DNA repair enzymes, such as 53BP1 and APE1, are endowed with RNA binding abilities. In this work, after reviewing the recent literature supporting a role of LLPS in DDR, we analyze, as a proof of principle, the interactome of APE1 using a bioinformatics approach to look for clues of LLPS in BER. Some of the APE1 interactors are associated with cellular processes in which LLPS has been either proved or proposed and are involved in different pathogenic events. This work might represent a paradigmatical pipeline for evaluating the relevance of LLPS in DDR.
\end{abstract}

Keywords: Base excision repair; bioinformatics; interactome; phase-separation; RNA.

\footnotetext{
*Corresponding author: Gianluca Tell, Laboratory of Molecular Biology and DNA repair, Department of Medicine, University of Udine, p.le M. Kolbe 4, 33100, Udine, Italy, E-mail: gianluca.tell@uniud.it Damiano Tosolini, Emiliano Dalla, Giulia Antoniali, Laboratory of Molecular Biology and DNA repair, Department of Medicine, University of Udine, p.le M. Kolbe 4, 33100, Udine, Italy
}

\section{Phase separation in nuclear organization and functions related to DNA damage response}

Nuclear dynamics, among other crucial cellular processes, has been recently established to be tuned, at least in part, by the widespread phenomenon of phase separation [1]. After a decade of active research, it is now accepted that this demixing process is a thermodynamicallydriven phenomenon, giving rise to a variety of dynamic bodies (i.e., biomolecular condensates, BMCs), primarily composed of nucleic acids and proteins [2] interacting through quinary interactions [3], frequently involving unstructured portions of proteins, especially intrinsically disordered regions (IDRs) [4,5]. These components are thought to be under the control of effective regulatory systems, through the action of a number of cellular factors which precisely tune the assembly and the de-aggregation of these bodies via post-translational modifications (PTMs), thus promoting a localized induction of condensates [6]. Some examples of nuclear processes proposed to be shaped by phase separation are heterochromatin domain formation, transcription, nucleolar metabolism, and DNA damage response (DDR). Indeed, it has been shown that chromatin structure dynamics may be regulated through phase-separation of several proteins (e.g., HP-1 and BRD4) involved in the reading of epigenetic marks on histone tails [7-9]; histone tail-DNA interactions might have a role in this process, as well [9]. An outstanding example of phase separation in the nucleus is represented by the nucleolus, the cellular body devoted to ribosome biogenesis. In recent years, it has been demonstrated that nucleoli may arise by phase separation induced by transcription of rRNAs from their genomic loci $[10,11]$ and that their three layers constitute an example of nested phase-separated domains [12]: indeed these domains, namely the fibrillar center, the dense fibrillar component and the granular component, 
each contributing to ribogenesis through different steps of rRNA maturation and ribosome assembling, although sharing the rRNA as a major phase separating agent and being in direct interaction, do not mix and preserve their functional specificity as a consequence of the biophysical properties of the components [12]. An additional process, for which a phase separation mechanism has been proposed, is represented by DNA transcription: recent studies have shed new light on the actual mechanism of recruitment of transcription factors, proposing cooperative kinetics to explain the effects driven by enhancers and super-enhancers, via demixing processes of transcription factors themselves [13,14]. However, transcription and other processes claimed to be driven by phase separation (e.g. heterochromatinization) remain to be fully characterised because they differ from biomolecular condensates in some aspects, which are reviewed in [1]. In particular, some compartments, characterized by many phase-separating features, do not strictly respect the canonical features of liquid biocondensates (namely the round shape, no shear elasticity, and internal dynamics), raising the question of whether phase-separation could be displayed in several different aspects. For example, paraspeckles, although regarded as demixed bodies upon NEAT1 increase, show a one-axis preferential growth, unusual for LLPS-based granules. Heterochromatic domains, instead, which were proposed to form by phase separation because of their apparent properties of coalescing, for excluding inert probes and for causing density transition in HP-1 distribution, were finally shown to undergo their round shape degeneration several cell cycles after phase separation occurred, thus denying the initial hypothesis [15].

\section{Role of RNA in promoting the recruitment of DNA repair enzymes at the lesion site}

RNA is an important element of biomolecular condensates, and some studies demonstrated its involvement in DNA repair. The first evidence of this involvement was provided by the DDR-related action of retrotransposons in yeast: indeed, it was shown that retrotransposon elements might replace homologous sequences and become integrated at the lesion site [16,17]. In [18], the authors suggested an additional link existing between retrotransposons and DDR: while reverse transcriptases could promote repair by canonical transcripts, integrases might promote cDNA insertion, and cDNA might act as a template to bridge the double-strand break (DSB), leading to the repair by "in trans" or "in cis" mechanisms. Additionally, with regard to the repair of DSBs, it was recently suggested that Rad52 might promote transcript-dependent DSB repair through inverse strand exchange, likely followed by reverse transcription of a ssDNA overhang [19-21]. This perspective is supported by the mounting evidence accounting for R-loops formation as a physiological regulator in the genome [22,23]. Notably, Rad52 (in yeast) and FUS (in human) were observed to contribute to the formation of a molecular biocondensate at DSB sites, carrying out different roles, namely the organization of nuclear microtubule filaments [24], protecting the resected end of lesions and promoting DNA-damage signaling $[25,26]$, as well as recruiting other DSB-repair-related enzymes [27]. Similarly, 53BP1, which is known to be important for DSB signaling and to affect the progression of the cell cycle, was demonstrated to localize to liquid compartments $[25,26]$.

Recently, it was found that ncRNAs seem to play a critical role in the formation of a liquid compartment at the DSB site. In detail, a novel class of RNAs has been defined and named DDRNAs: they are produced from the processing of dilncRNAs (damage-induced long noncoding RNAs), which are transcribed at DSBs foci in a bidirectional manner $[28,29]$. DDRNAs are guided to the lesion site by dilncRNAs and both of them are supposed to contribute to the recruitment of repair enzymes $[28,30]$. Additionally, examples of the active role of lncRNAs promoting, in trans, the recruitment of the DSB repair machinery in a demixing-mediated manner have been described. The lncRNA LINP1, for example, allows the formation of a liquid department where Ku70 and Ku80 can demix to effectively accomplish non-homologous end joining (NHEJ) repair [31]. In addition, other ncRNAs have also been associated with DDR signaling [32] and cellwide effects [33,34].

A typical example showing the involvement of RNAmoiety in DDR is PARylation (and Mono ADP-ribosylation, MARylation), a post-translational modification consisting in the addition of single or multiple ADP-ribose molecules to both proteins and DNA [35]. These modifications are introduced by the PARP enzymes family and represent one of the main signals of genomic damage in cells $[36,37]$. In particular, PARP enzymes are now known to catalyze the addition of MAR or PAR moieties at single-strand break (SSB) and DSB loci, thus mediating the signaling of those damaging events [35,38]. These modifications are also known to direct the formation of damaged DNA-enriched compartments and to recruit demixing factors, like FUS 
[39,40]. To date, albeit it is known that: i) PARP interacts with several BER factors (e.g., XRCC1, POL $\beta$, and LIG3); ii) PARP modulates the activity of glycosylases [41,42] and the 3'-exonuclease activity of APE1 [43] and that iii) PARP inhibition significantly hampers the efficiency of the BER pathway [44,45], there is no evidence for the formation of a demixed compartment hosting the BER-mediated DDR.

\section{Interactomes of DNA repair enzymes: focus on RNA processing proteins}

Along with the involvement of RNA molecules themselves, it is widely accepted that the interaction with RNA represents a key feature of most DDR enzymes, both in direct and indirect manners. It was shown that many RNA binding proteins (RBPs) are required to ensure proper production of DDR factors (as reviewed in [46]) via posttranslational regulation of their transcripts, allowing them to escape the general translational repression occurring upon DNA damage and thus indirectly influencing the repair process. Nonetheless, RBPs were shown to directly take part in DDR, since enzymes involved in mRNA and miRNA processing have been associated with DNA repair. For instance, RBM14 is a RBP involved in alternative splicing and it is recruited to DSB sites via PARP1 [47,48]; likewise, HNRNPD is necessary for the DNA resection step in the homologous recombination pathway [49]. Helicases, for example DEAD-box helicases, are interesting RNAinteracting proteins involved in RNA metabolism [50,51] and in DDR [52,53] and some of them were shown to participate in demixing bodies $[54,55]$.

Interestingly, the small non-coding RNA machinery, including DICER and DROSHA, is also important for DSB repair, since its products appear to be fundamental in recruiting some repairing factors $[29,56]$.

These observations collectively suggest that a strong involvement in RNA metabolism is common among phase separating factors acting in DDR and that this might be a key feature to be investigated.

\section{The Apurinic/Apyrimidinic Endonuclease (APE1) is a crucial BER enzyme}

The Apurinic/Apyrimidinic Endonuclease (APE1) is a central enzyme in the BER pathway, acting as the main
AP-endonuclease in mammalians [57]. However, this enzyme was recently characterized as possessing many non-canonical functions (Figure 1) associated with RNA metabolism, including taking part in the biogenesis of ncRNAs (possibly through the interaction with DROSHA [58] or functionally interacting with other phase separating factors, like NPM-1 during rRNA biogenesis) [59], as well as with several RNA species [60]. These novel functions, partly supported by the capability of APE1 to bind to different nucleic acids, tremendously expand its functions toward the RNA world, which is clearly connected to LLPS. The ability of APE1 to bind and process RNA seems to be empowered by the disordered $\mathrm{N}$-terminus of this protein, which is thought to be a recent evolutionary acquisition in mammals and it is found to be highly conserved in mammals, possibly constituting an important gain of function example [61]. This domain, in fact, was found to be essential for APE1 recruitment to nuclear subcompartments, including the nucleolus [62], and for its interaction with other BER factors [63] and other partners like NPM1 [61], which, in recent years, have also been linked to several novel functions including LLPS [64].

It is still not known whether the unstructured domain of APE1 and its RNA-binding abilities might represent an evolutionary gain of function to promote BER recruitment and to coordinate the action of the different enzymes, considering that every intermediate reaction product (i.e., the abasic site generated by glycosylases, the nick generated by APE1, etc.) results even more toxic than the original lesion processed by BER itself. This is particularly important, given that APE1 is much more abundant than all the other BER proteins and, in tumor cells, it is highly overexpressed. Therefore, the assumption that APE1 is only required for DNA repair by BER is somehow too limited.

\section{Bioinformatics analysis of demixing proteins: the case study of the APE1 interactome suggests a novel hypothesis for triggering the Base Excision Repair pathway}

The aforementioned features define APE1 as a reasonable candidate for a preliminary investigation questioning the involvement of a LLPS mechanism in its recruitment. Although there are other DDR-related, BER-involved 


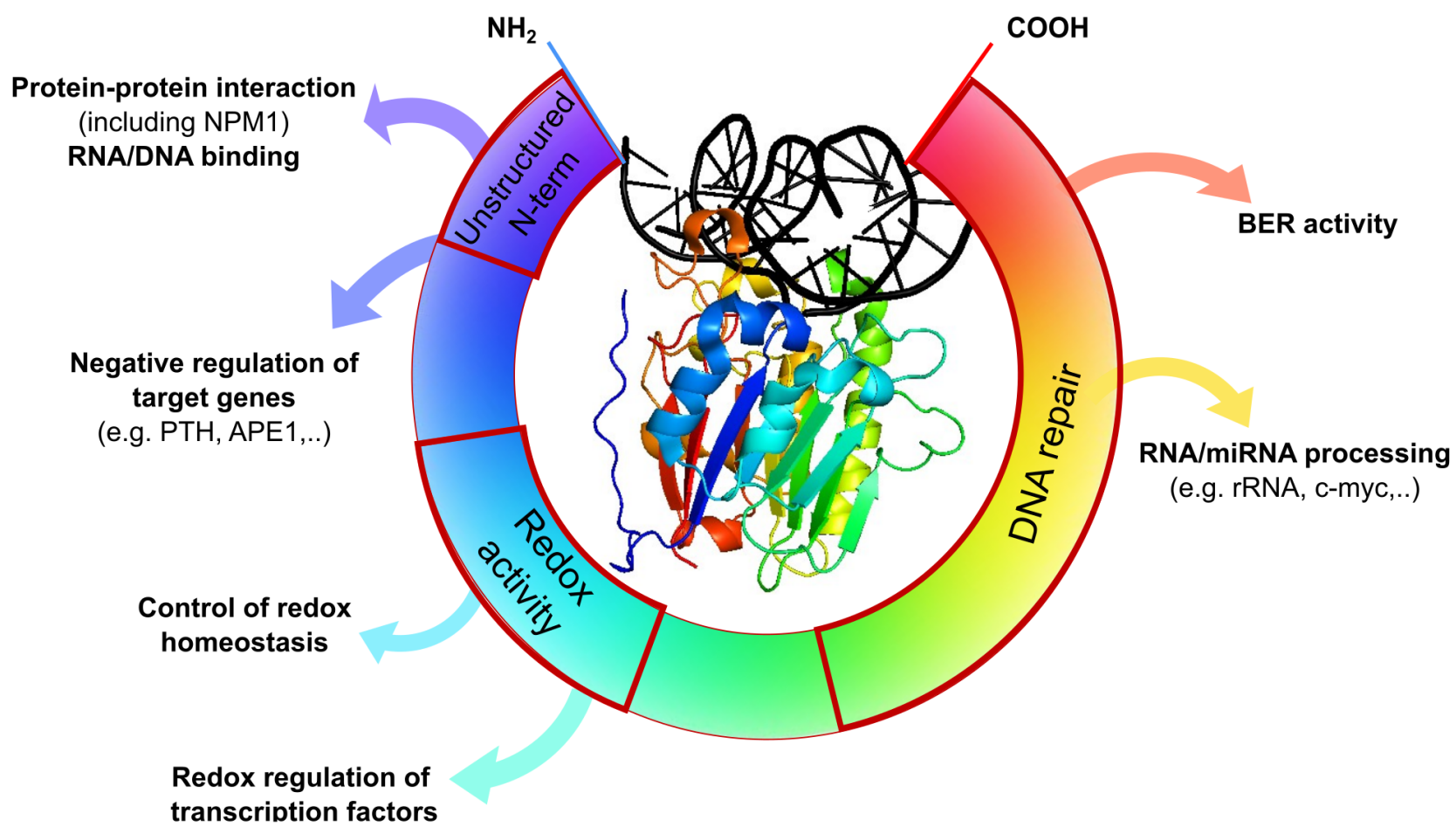

Figure 1: Main APE1 functions. The main functions of APE1 are mapped to the primary structure portions responsible for such effects $[58,73,89]$. The APE1 crystal structure bound to abasic DNA is displayed starting from the PDB deposited structure (6W0Q, [90]) and was modified using PyMOL software. Rainbow colors: APE1 sequence; black: substrate DNA.

proteins, known for being central for the recruitment of other factors and exhibiting even multiple IDRs (e.g., XRCC1), nevertheless APE1 represents a particularly interesting subject because of its role in RNA metabolism.

Here, we propose a bioinformatics approach that might be employed to obtain some useful insights on the LLPS world, defining a pipeline to help to direct the following experimental activity. This pipeline employs the APE1 interactome we recently defined [65]. This pool of interactors was assessed by an unbiased pull-down approach, performed taking advantage of a FLAG-tagged APE1 recombinant form, followed by protein complexes characterization through a MS/MS approach. In that work, which allowed us to identify almost 500 APE1-PPIs, we intentionally used a non-targeted approach to identify components involved in direct APE1-protein interactions, as well as molecules whose interaction with APE1 is indirectly mediated by RNA/DNA or other proteins. This approach, very stringent in order to avoid misleading identification of protein complex elements, might enhance the identification of cofactors colocalizing with APE1 in biomolecular condensates, making this analysis more suited for BMCs applications. In fact, the multiple biological functions attributed to APE1 and its localization in various subcellular districts further expand the list of its possible protein binding partners, as deriving from direct or indirect interactions. The condition we observed for APE1 is similar to the one recently reported for two APE1-binding partners, namely XRCC6 and XRCC5, which have been similarly demonstrated binding to RNA/DNA and to about 300 proteins [66], or previously described for proteins present in chaperone machineries [67]. Other examples of proteins having hundreds of interactors (as deduced by a single immunocapture experiment) are already present in the scientific literature [68-70]. At the same time, we were aware of the relevance of the lack of specificity for ascertaining direct PPIs in the methodology we used. Nevertheless, this approach is similar to others already used in previous papers allowing the identification of original APE1-binding partners [59,71,72]. It is finally to be noted that about 100 APE1-PPIs (among the ones reported in this study) were already demonstrated to be real APE1-PPIs in previous studies from us or other research groups; important examples in this context are NPM1, SFPQ and hnRNPK, as proved by independent binding and functional assays [59,71,73,74].

In the first step of our proposed bioinformatics analysis, we retrieved the disorder content of APE1-PPIs 
from the MobiDB database (version 3.1.0) [75], which integrates manual reviews and in silico predictions. Out of 515 interactors, we were able to retrieve data for about 350 proteins (for the remaining proteins, the disorder index was not computed). We examined the distribution of these values and most of the interactors were characterized by a low disorder content (below 0.2) (Figure 2A).

Then, we collected the APE1-PPI data from PhaSePro [76], a manually curated collection of proteins characterized as demixing in vivo. The output consisted of seven APE1 interactors, namely APP, NPM1, LGALS3, HNRNPA1, FUS, SFPQ, and ESR1, fully characterized as demixing by ad hoc experiments. Their disorder content was pointed out as a reference in the general distribution (Figure 2A).

As a control, we also evaluated the disorder content of all the proteins profiled in the PhaSePro database for LLPS, focusing in particular on characterizing the minimum value required for partitioning and the dependence, if any, on demixing partners. Interestingly, we could define two different groups (Figure 2B): one, including the majority of the examined proteins, which showed a progressive increase in disorder content starting from about 0.2 and ramping up to 0.9 and a second, much smaller, centered on 0.05. To our surprise, the feature that mainly differentiated the latter was the requirement of a demixing partner, TIA1 representing the only exception; on the contrary, less than one-third of the proteins belonging to the first group behaved in the same way, with LAT curiously characterized by having the highest disorder content but also the necessity of a demixing partner.

Considering the two distributions, we noticed that all the reviewed demixing proteins known for not requiring additional partners were basically characterized by an internal disorder content greater than 0.15; thus, we focused on the APE1 PPI having a disordered content above that threshold, defining a subset composed of 88 members. We compared them to entries in PhaSepDB [77], which aggregates a wide range of direct and indirect evidence of proteins phase separation (e.g., fully demonstrated or just suggested by high-throughput data), defining a final set of 49 likely demixing interactors (Table 1).

To gain some insights on the biological processes involving these proteins, we performed a functional enrichment analysis [78] employing ClueGO [79], a Cytoscape [80] plugin allowing to use different ontologies/ databases, focusing on biological processes (Figure 3A) and intracellular localization (Figure 3B).
We first analyzed the set of 49 likely demixing interactors defined by PhaSepDB, and the results highlighted 34 significantly enriched terms associated with six major processes by ClueGO, as shown in Figure 3A. Interestingly, most of these terms were associated with gene expression and RNA processing, while the rest was associated with viral and telomeric regulation.

A second analysis of the same gene set took into consideration the intracellular localization. We obtained enriched terms related to euchromatin, spliceosome, and translation preinitiation complex, cellular departments strictly linked to the metabolism of nucleic acids that, hence, might act as dynamic scaffolds for liquid-like structures (Figure 3B).

We repeated the same analysis on the seven interactors fully characterized as demixing by PhaSePro, adding APE1 as the ideal center of the functional network. Interestingly, one of the three enriched terms (Figure 4) pointed to the formation of amyloids, common hallmarks of neurodegenerative diseases, which have been related to liquid-demixing proteins $[81,82]$.

Lastly, we compared the list of 49 interactors to the MSigDB database, a collection of gene sets co-expressed and/or involved in physiological and pathological processes (i.e., molecular signatures) obtained by a data mining approach that functionally complements the ontologies previously investigated using ClueGO $[83,84]$. For this investigation, the $\mathrm{H}, \mathrm{C} 4, \mathrm{C} 6$ and $\mathrm{C} 7$ collections of MSigDB were chosen as terms of comparison. These signatures have different origins and meanings: the $\mathrm{H}$ collection is made of signatures characterizing welldefined biological processes, C4 gathers cancer-related signatures originated by data mining of large microarray data, C6 collects signatures related to pathways deregulated in cancer while $\mathrm{C} 7$ collects signatures related to the immune system and its deregulation. A false discovery rate (FDR) threshold was set to 0.05 to establish significant results (Benjamini-Hochberg correction implemented by MSigDB). We took into account the first 50 enriched results (Table 2).

We obtained a significant association with different kinds of cancer signatures, namely: liver, prostate, and hematological tumors; furthermore, additional signatures were also linked to the activation of the PBMCs (peripheral blood mononucleated cells). Finally, enriched terms also pointed to general biological processes such as ribogenesis, protein biosynthesis, and mRNA splicing, consistently with previous ClueGO results. These terms strongly suggest the existence of a relationship, both physical and functional, with: i) nucleic acids (especially 
A

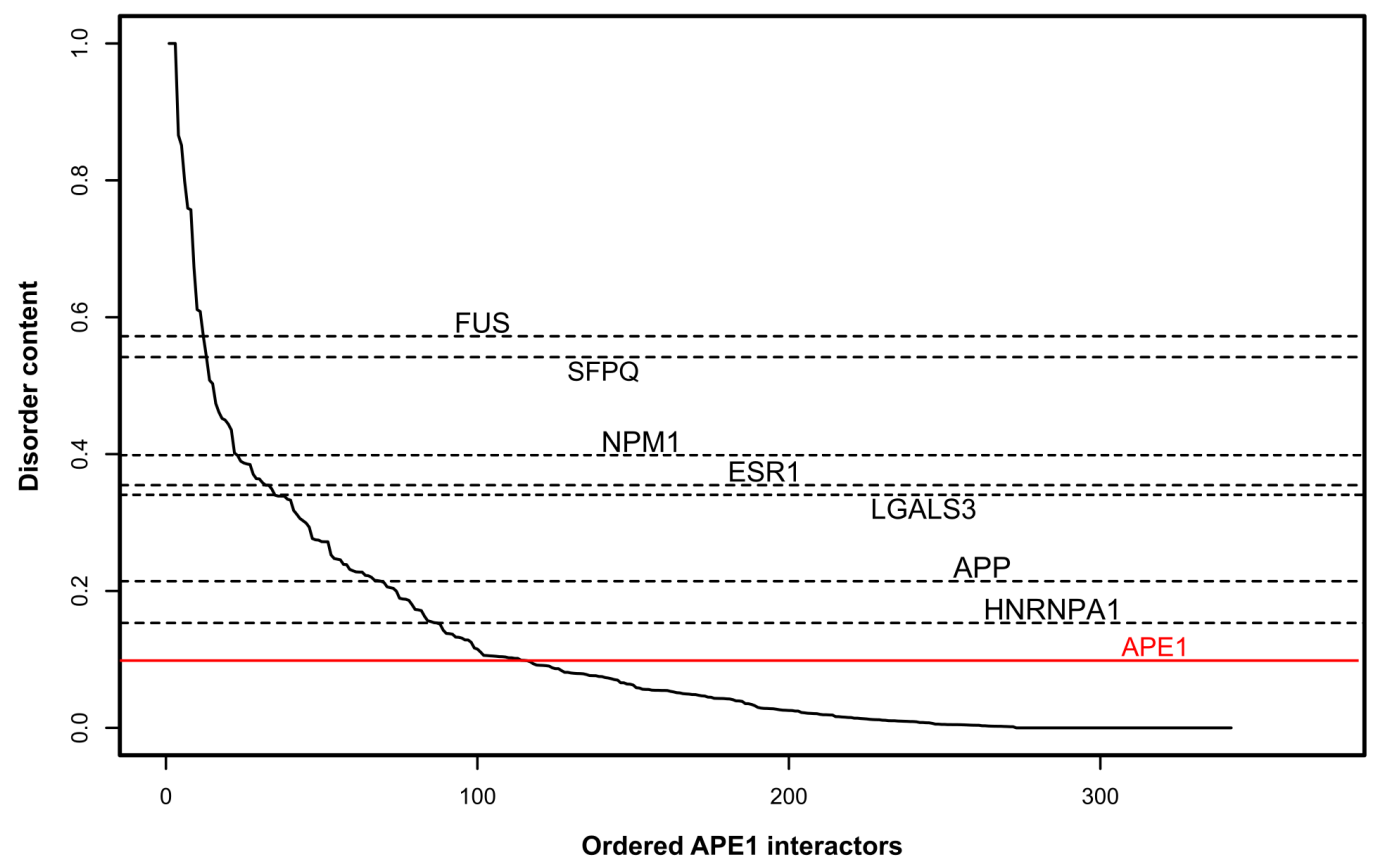

B

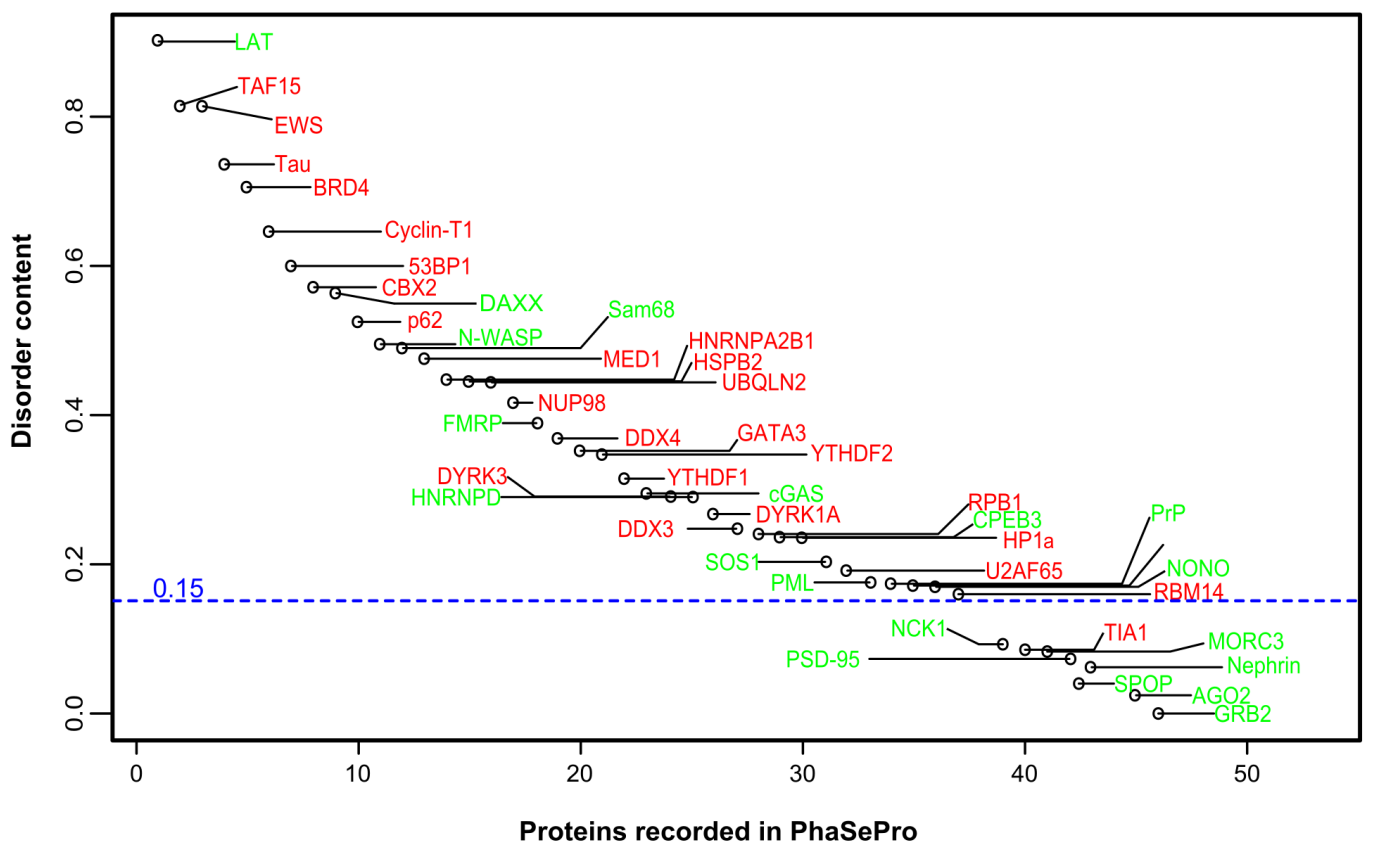

Figure 2: Distribution of the examined proteins disorder content. (A) APE1 protein interactors, ordered by decreasing disorder content as retrieved from MobiDB, are identified by their position in the list of interactors. Dashed, horizontal lines highlight the position of the APE1 interactors known to undergo phase-separation according to PhaSePro. APE1 level of disorder is also highlighted by a red, solid line. (B) PhaSePro proteins, ordered by decreasing disorder content as retrieved from MobiDB, are colored according to the necessity (green) or independence (red) for a demixing partner. The blue dashed line highlights the 0.15 threshold in disorder content used to select proteins for functional characterization. 
Table 1: List of the 49 APE1 interactors likely to undergo phase separation. These 49 interactors were identified by comparing the APE1 PPI network to the LLPS-oriented databases PhaSepDB and PhaSePro and characterized by an internal disorder content index (as computed by MobiDB) greater or equal to 0.15 . Their UniProtID and the internal disorder content index are reported.

\begin{tabular}{lll}
\hline & UniProtIDs & Disorder content \\
\hline ANP32E & Q9BTT0 & 0.358 \\
CBX3 & Q13185 & 0.306 \\
DYNC1H1 & Q14204 & 0.171 \\
EIF3G & 075821 & 0.272 \\
EIF3J & 075822 & 0.461 \\
EIF3L & Q9Y262 & 0.339 \\
EIF4B & P23588 & 0.851 \\
FUBP1 & Q96AE4 & 0.339 \\
FUS & P35637 & 0.572 \\
GTF2I & P78347 & 0.212 \\
HDAC1 & Q13547 & 0.220 \\
HDAC2 & Q92769 & 0.205 \\
HMGA1 & P17096 & 0.673 \\
HNRNPA1 & P09651 & 0.153 \\
HNRNPC & P07910 & 0.474 \\
HNRNPK & P61978 & 0.253 \\
HNRNPL & P14866 & 0.333 \\
HNRNPUL1 & Q9BUJ2 & 0.450 \\
ILF2 & Q12905 & 0.154 \\
ILF3 & Q12906 & 0.385 \\
LASP1 & Q14847 & 0.303 \\
LGALS3 & PAP2K1 & 0.340 \\
\hline MDM2 & P1793 & \\
\hline
\end{tabular}

\begin{tabular}{lll}
\hline & UniProtIDs & Disorder content \\
\hline NPM1 & P06748 & 0.398 \\
NUDC & Q9Y266 & 0.230 \\
NUDT3 & 095989 & 0.215 \\
RBMX & P38159 & 0.757 \\
RCC2 & Q9P258 & 0.172 \\
RNF4 & P78317 & 0.163 \\
RPL14 & P50914 & 0.349 \\
RPS3A & P61247 & 0.189 \\
RPSA & P08865 & 0.247 \\
SFPQ & P23246 & 0.542 \\
SIRT1 & Q96EB6 & 0.364 \\
SNRPA & P09012 & 0.277 \\
SNRPA1 & P09661 & 0.318 \\
SNRPB2 & P08579 & 0.222 \\
SRP14 & P37108 & 0.272 \\
SRPK1 & Q96SB4 & 0.275 \\
SRPK2 & P78362 & 0.371 \\
SYNCRIP & 060506 & 0.204 \\
TCEA1 & P23193 & 0.435 \\
TERF1 & P54274 & 0.312 \\
TRA2B & P62995 & 0.611 \\
TRIM28 & Q13263 & 0.216 \\
YBX1 & P67809 & 0.759 \\
ZC3HAV1 & Q722W4 & 0.186 \\
\hline & & \\
\hline
\end{tabular}

RNA); ii) their metabolism, and iii) the compartments in which they accumulate.

We hypothesize that this connection might occur through direct or indirect recruitment to liquid demixing bodies, the impairment of which could be related to several pathological conditions. This particular association with nucleic acids might also represent evidence for a novel APE1 function related to phase-separation: on such a basis, more investigation is demanded to elucidate the possible role of biocondensates in BER pathway triggering, that could explain the role of these RNA-interacting partners, and could also be of interest for establishing novel protocols for drug development.

\section{Concluding remarks}

This review provides several pieces of evidence suggesting the involvement of new mechanisms and new experimentally validated protein candidates belonging to 
A

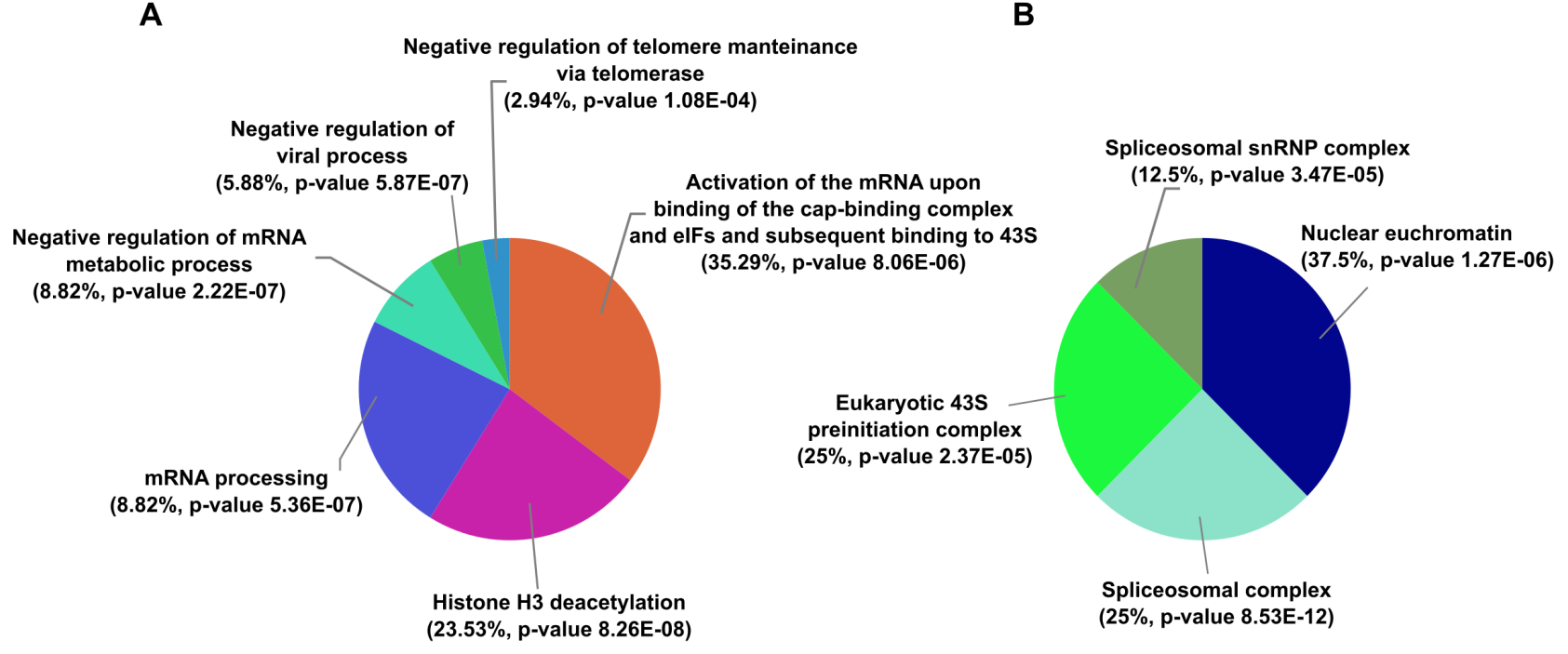

Figure 3: Functional enrichment analysis of the set of 49 interactors likely correlated with phase separation. For each analysis, ClueGO identified a panel of enriched terms (adjusted pValue<0.05) that were clustered. (A) Clusters of enriched terms associated with biological processes involving APE1 likely demixing interactors (Queried databases: WikiPathways [66], KEGG [67], CORUM [68], ClinVar [69], Reactome [70], GOBiologicalProcess [71]). (B) Clusters of enriched terms (adjusted pValue<0.05) associated with subcellular localization (Queried database: GOCellularComponent [71]). Percent values refer to the amount of enriched terms associated with each cluster. P-values are referred to the clusters.

Amyloid-beta formation (33.33\%, p-value 2.00E-06)

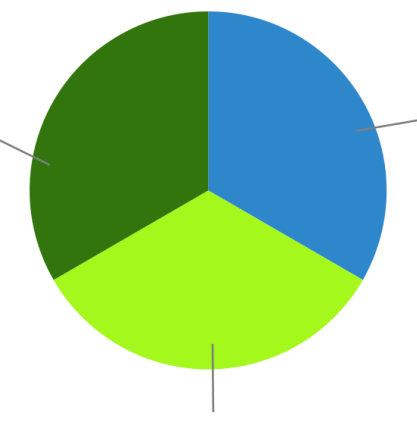

Advanced glycosilation and product receptor signaling (33.33\%, p-value 1.35E-05)
Transcriptional regulation by AP-2

(TFAP") family of transcription factors

(33.33\%, p-value 8.08E-05)

Figure 4: Functional enrichment analysis of the extended set of PhaSePro characterized proteins (including APE1). Enriched biological processes (adjusted pValue<0.05) interestingly suggest a role in amyloid formation, regulation of transcription and AGE receptor signaling (Queried databases: WikiPathways, KEGG, CORUM, ClinVar, Reactome, GOBiologicalProcess. Minimum number of genes per cluster set to 2).

the APE1 interactome in phase separating events, although the underlying direct or indirect molecular mechanisms are still to be demonstrated.

The results here obtained, through the proposed bioinformatics pipeline, would be useful in further experimental validation to identify demixing cofactors that might be crucial in reproducing phase-separation in vitro [85]. For example, on the basis of the close relationship existing between APE1 and NPM1, the consequential follow up of this investigation could be represented by exploring the possible joint phase-separation of these proteins in the presence of rRNA, which is known to be required for NPM1 phase separation and that might also be necessary to APE1 demixing. Moreover, drugs selectively impairing LLPS might be employed to validate this hypothesis: 1,6-hexanediol, for example, has been already used in previous works to show the demixed status of some bodies. Some doubts have been raised about this proving technique since this molecule might produce artifacts in living cells; in fact, its amphipathic nature is not able to 
Table 2: The 50 most significantly enriched molecular signatures determined by the MSigDB molecular signature enrichment analysis. The name of the molecular signatures is provided, along with their False Discovery Rate (FDR) as computed by GSEA.

\begin{tabular}{ll}
\hline Molecular Signatures & FDR \\
\hline Genes in the cancer module 32. & $4.13 \mathrm{E}-32$ \\
Neighborhood of ANP32B & $8.23 \mathrm{E}-28$ \\
Neighborhood of ACP1 & $2.31 \mathrm{E}-25$ \\
Neighborhood of G22P1 & $5.21 \mathrm{E}-25$ \\
Neighborhood of FBL & $2.39 \mathrm{E}-22$ \\
Neighborhood of DEK & $6.29 \mathrm{E}-22$ \\
Neighborhood of RAN & $6.55 \mathrm{E}-22$ \\
Neighborhood of GNB1 & $5.39 \mathrm{E}-21$ \\
Neighborhood of HDAC1 & $1.76 \mathrm{E}-20$ \\
A subgroup of genes regulated by MYC - version 1 & $3.04 \mathrm{E}-20$ \\
(v1). & \\
Neighborhood of EIF3S6 & $2.53 \mathrm{E}-19$ \\
Neighborhood of UBE2I & $3.64 \mathrm{E}-19$ \\
Neighborhood of CSNK2B & $1.84 \mathrm{E}-18$ \\
Neighborhood of RAD23A & $1.84 \mathrm{E}-18$ \\
Neighborhood of FBL & $2.3 \mathrm{E}-18$ \\
Neighborhood of ANP32B & $1.53 \mathrm{E}-16$ \\
Neighborhood of BUB3 & $1.59 \mathrm{E}-16$ \\
Neighborhood of CCNI & $6.52 \mathrm{E}-15$ \\
Neighborhood of APEX1 & $9.84 \mathrm{E}-15$ \\
Neighborhood of CSNK2B & $1.2 \mathrm{E}-14$ \\
Genes in the cancer module 98. & $1.72 \mathrm{E}-14$ \\
Genes in the cancer module 198. & \\
Neighborhood of NPM1 & \\
\hline Genes in the cancer module 83.14 \\
\hline
\end{tabular}

impair some of the most common molecular interactions giving rise to BMCs.

We suggest that our approach may contribute to uncovering new molecular strategies for the therapy of human diseases that have been recently linked to phase partitioning, especially neurodegenerative diseases (e.g., Alzheimer's disease, amyotrophic lateral sclerosis (ALS), frontotemporal dementia). In fact, well known ALS-related mutations, such as substitutions in the mostly disordered C-terminus of TDP-43 and the hexanucleotide expansion in C9orf72, were shown to impair phase separation of

\begin{tabular}{ll}
\hline Molecular Signatures & FDR \\
\hline Neighborhood of ACTG1 & $1.58 \mathrm{E}-13$ \\
Genes up-regulated in polymorphonuclear leukocytes & $3.46 \mathrm{E}-13$ \\
(24h): control versus infection by A. phagocytophilum & \\
Neighborhood of ACTG1 & $4.38 \mathrm{E}-13$ \\
Neighborhood of SMC1L1 & $2.73 \mathrm{E}-12$ \\
RNA splicing. & $3.93 \mathrm{E}-12$ \\
Neighborhood of TDG & $4.72 \mathrm{E}-12$ \\
Neighborhood of APEX1 & $4.88 \mathrm{E}-12$ \\
Neighborhood of ERH & $5.13 \mathrm{E}-12$ \\
Neighborhood of SOD1 & $9.97 \mathrm{E}-12$ \\
Neighborhood of XRCC5 & $1.12 \mathrm{E}-11$ \\
Neighborhood of EIF4A2 & $2.45 \mathrm{E}-11$ \\
Neighborhood of PCNA & $2.45 \mathrm{E}-11$ \\
Neighborhood of PSME1 & $2.9 \mathrm{E}-11$ \\
Genes in the cancer module 151. & $3.92 \mathrm{E}-11$ \\
Neighborhood of UBE2N & $2.8 \mathrm{E}-10$ \\
TFs and nuclear. & $2.95 \mathrm{E}-10$ \\
Protein biosynthesis and ribosomes. & $3.01 \mathrm{E}-10$ \\
Neighborhood of EIF3S2 & $3.48 \mathrm{E}-10$ \\
\hline Neighborhood of DEK & $7.02 \mathrm{E}-11$ \\
Neighborhood of HAT1 & $7.07 \mathrm{E}-11$ \\
Neighborhood of HDAC1 & $9.7 \mathrm{E}-11$ \\
Neighborhood of ST13 & $1.46 \mathrm{E}-10$ \\
Neighborhood of NPM1 & $1.74 \mathrm{E}-10$ \\
Neighborhood of DDX5 & $1.99 \mathrm{E}-10$ \\
& \\
\hline &
\end{tabular}

these proteins, suggesting their relevance in the onset of the pathological condition $[86,87]$. Additionally, altered proteostasis, which leads to the formation of aggregates in neuronal cells, is a hallmark of such conditions and was related to phase separation. Nonetheless, a complete understanding of how this aggregation influences the pathological outcome is still missing and requires further investigation to elucidate the exact relationship linking the fiber formation and the toxic effect [88]. Another interesting question, arising from the hypothesis of pathological misregulation of liquid compartments, 
concerns how these bodies could be physiologically maintained and the impaired regulatory mechanisms leading to irreversible aggregation, if any [88]. Lastly, since anti-cancer therapies are partly based on inefficient DNA repair, further characterization of the molecular mechanisms and the associated dynamics, along with the advanced unfolding of the interactomes involved in such pathways, might uncover new oncological targets. Therefore, the uncovering of a possible LLPS-related mechanism would certainly improve our knowledge on how to target deregulated processes of the DDR, selectively impacting on several human pathologies.

Author contributions: GT designed the paper outline and the research plan. DT performed the analysis. GA and ED analyzed data. DT, GA and ED wrote the manuscript. GT supervised the writing of the whole manuscript.

Acknowledgments: The research leading to these results has received funding from AIRC under IG 2017 - ID. 19862 project - P.I. Tell Gianluca.

Conflict of interests: Authors state no conflict of interest.

\section{References}

1. A P, Weber SC. Evidence for and against Liquid-Liquid Phase Separation in the Nucleus. Noncoding RNA. 2019 Nov;5(4):50.

2. Banani SF, Lee HO, Hyman AA, Rosen MK. Biomolecular condensates: organizers of cellular biochemistry. Nat Rev Mol Cell Biol. 2017 May;18(5):285-98.

3. Ribeiro S, Ebbinghaus S, Marcos JC. Protein folding and quinary interactions: creating cellular organisation through functional disorder. FEBS Lett. 2018 Sep;592(18):3040-53.

4. Protter DS, Rao BS, Van Treeck B, Lin Y, Mizoue L, Rosen $M K$, et al. Intrinsically Disordered Regions Can Contribute Promiscuous Interactions to RNP Granule Assembly. Cell Rep. 2018 Feb;22(6):1401-12.

5. Lin Y, Protter DS, Rosen MK, Parker R. Formation and Maturation of Phase-Separated Liquid Droplets by RNA-Binding Proteins. Mol Cell. 2015 Oct;60(2):208-19.

6. Söding J, Zwicker D, Sohrabi-Jahromi S, Boehning $M$, Kirschbaum J. Mechanisms for Active Regulation of Biomolecular Condensates. Trends Cell Biol. 2020 Jan;30(1):4-14.

7. Erdel F, Rippe K. Formation of Chromatin Subcompartments by Phase Separation. Biophys J. 2018 May;114(10):2262-70.

8. Sanulli S, Trnka MJ, Dharmarajan V, Tibble RW, Pascal BD, Burlingame AL, et al. HP1 reshapes nucleosome core to promote phase separation of heterochromatin. Nature. 2019 Nov;575(7782):390-4.

9. Gibson BA, Doolittle LK, Schneider MW, Jensen LE, Gamarra $\mathrm{N}$, Henry L, et al. Organization of Chromatin by Intrinsic and Regulated Phase Separation. Cell. 2019 Oct;179(2):470-484. e21.
10. Smirnov E, Borkovec J, Kováčik L, Svidenská S, Schröfel A, Skalníková M, et al. Separation of replication and transcription domains in nucleoli. J Struct Biol. 2014 Dec;188(3):259-66.

11. Falahati H, Pelham-Webb B, Blythe S, Wieschaus E. Nucleation by rRNA Dictates the Precision of Nucleolus Assembly. Curr Biol. 2016 Feb;26(3):277-85.

12. Feric M, Vaidya N, Harmon TS, Mitrea DM, Zhu L, Richardson TM, et al. Coexisting Liquid Phases Underlie Nucleolar Subcompartments. Cell. 2016 Jun;165(7):1686-97.

13. Sabari BR, Dall'Agnese A, Boija A, Klein IA, Coffey EL, Shrinivas $\mathrm{K}$, et al. Coactivator condensation at super-enhancers links phase separation and gene control. Science (80-) . 2018;361(6400):eaar3958. https://doi.org/10.1126/science. aar3958.

14. Nair SJ, Yang L, Meluzzi D, Oh S, Yang F, Friedman MJ, et al. Phase separation of ligand-activated enhancers licenses cooperative chromosomal enhancer assembly. Nat Struct Mol Biol. 2019 Mar;26(3):193-203.

15. Strom AR, Emelyanov AV, Mir M, Fyodorov DV, Darzacq X, Karpen $\mathrm{GH}$. Phase separation drives heterochromatin domain formation. Nature. 2017 Jul;547(7662):241-5.

16. Moore JK, Haber JE. Capture of retrotransposon DNA at the sites of chromosomal double-strand breaks. Nature. 1996 Oct;383(6601):644-6.

17. Morrish TA, Gilbert N, Myers JS, Vincent BJ, Stamato TD, Taccioli $\mathrm{GE}$, et al. DNA repair mediated by endonuclease-independent LINE-1 retrotransposition. Nat Genet. 2002 Jun;31(2):159-65.

18. Meers C, Keskin H, Storici F. DNA repair by RNA: Templated, or not templated, that is the question. DNA Repair (Amst). 2016 Aug;44:17-21.

19. McDevitt S, Rusanov T, Kent T, Chandramouly G, Pomerantz RT. How RNA transcripts coordinate DNA recombination and repair. Nat Commun. 2018 Mar;9(1):1091.

20. Storici F, Bebenek K, Kunkel TA, Gordenin DA, Resnick MA. RNAtemplated DNA repair. Nature. 2007 May;447(7142):338-41.

21. Keskin H, Shen Y, Huang F, Patel M, Yang T, Ashley K, et al. Transcript-RNA-templated DNA recombination and repair. Nature. 2014 Nov;515(7527):436-9.

22. Niehrs C, Luke B. Regulatory R-loops as facilitators of gene expression and genome stability. Nat Rev Mol Cell Biol. 2020 Mar;21(3):167-78.

23. Tan J, Duan M, Yadav T, Phoon L, Wang X, Zhang JM, et al. An R-loop-initiated CSB-RAD52-POLD3 pathway suppresses ROS-induced telomeric DNA breaks. Nucleic Acids Res. 2020 Feb;48(3):1285-300.

24. Oshidari R, Huang R, Medghalchi M, Tse EY, Ashgriz N, Lee HO, et al. DNA repair by Rad52 liquid droplets. Nat Commun. 2020 Feb;11(1):695.

25. Kilic S, Lezaja A, Gatti M, Bianco E, Michelena J, Imhof R, et al. Phase separation of 53BP1 determines liquid-like behavior of DNA repair compartments. EMBO J. 2019 Aug;38(16):e101379.

26. Mirza-Aghazadeh-Attari $M$, Mohammadzadeh A, Yousefi B, Mihanfar A, Karimian A, Majidinia M. 53BP1: A key player of DNA damage response with critical functions in cancer. DNA Repair (Amst). 2019 Jan;73(73):110-9.

27. Lenken SC, Levone BR, Filosa G, Antonaci M, Conte F, Kizilimark $C$, et al. FUS-dependent phase separation initiates doublestrand break repair. bioRxiv. 2019; (preprint posted Oct. 11th, 2019). 
28. Pessina F, Giavazzi F, Yin Y, Gioia U, Vitelli V, Galbiati A, et al. Functional transcription promoters at DNA double-strand breaks mediate RNA-driven phase separation of damage-response factors. Nat Cell Biol. 2019 Oct;21(10):1286-99.

29. Francia S, Cabrini M, Matti V, Oldani A, d’Adda di Fagagna F. DICER, DROSHA and DNA damage response RNAs are necessary for the secondary recruitment of DNA damage response factors. J Cell Sci. 2016 Apr;129(7):1468-76.

30. Durut N, Mittelsten Scheid 0 . The Role of Noncoding RNAs in Double-Strand Break Repair. Front Plant Sci. 2019 Sep;10(September):1155.

31. Thapar R, Wang JL, Hammel M, Ye R, Liang K, Sun C, et al. Mechanism of efficient double-strand break repair by a long non-coding RNA. Nucleic Acids Res. 2020 Nov;48(19):10953-72.

32. Zhao K, Wang $X$, Xue X, Li L, Hu Y. A long noncoding RNA sensitizes genotoxic treatment by attenuating ATM activation and homologous recombination repair in cancers. PLoS Biol. 2020 Mar;18(3):e3000666.

33. Huarte $M$, Guttman $M$, Feldser D, Garber M, Koziol MJ, Kenzelmann-Broz D, et al. A large intergenic noncoding RNA induced by $\mathrm{p} 53$ mediates global gene repression in the $\mathrm{p} 53$ response. Cell. 2010 Aug;142(3):409-19.

34. Hung T, Wang Y, Lin MF, Koegel AK, Kotake Y, Grant GD, et al. Extensive and coordinated transcription of noncoding RNAs within cell-cycle promoters. Nat Genet. 2011 Jun;43(7):621-9.

35. Zarkovic G, Belousova EA, Talhaoui I, Saint-Pierre C, Kutuzov $M M$, Matkarimov BT, et al. Characterization of DNA ADPribosyltransferase activities of PARP2 and PARP3: new insights into DNA ADP-ribosylation. Nucleic Acids Res. 2018 Mar;46(5):2417-31.

36. Schreiber V, Dantzer F, Ame JC, de Murcia G. Poly(ADP-ribose): novel functions for an old molecule. Nat Rev Mol Cell Biol. 2006 Jul;7(7):517-28.

37. Gupte R, Liu Z, Kraus WL. PARPs and ADP-ribosylation: recent advances linking molecular functions to biological outcomes. Genes Dev. 2017 Jan;31(2):101-26.

38. Matta E, Kiribayeva A, Khassenov B, Matkarimov BT, Ishchenko AA. Insight into DNA substrate specificity of PARP1-catalysed DNA poly(ADP-ribosyl)ation. Sci Rep. 2020 Feb;10(1):3699.

39. Leung AK. Poly(ADP-ribose): A Dynamic Trigger for Biomolecular Condensate Formation. Trends Cell Biol. 2020 May;30(5):37083.

40. Singatulina AS, Hamon L, Sukhanova MV, Desforges B, Joshi V, Bouhss A, et al. PARP-1 Activation Directs FUS to DNA Damage Sites to Form PARG-Reversible Compartments Enriched in Damaged DNA. Cell Rep. 2019 May;27(6):1809-1821.e5.

41. Polo LM, Xu Y, Hornyak P, Garces F, Zeng Z, Hailstone R, et al. Efficient Single-Strand Break Repair Requires Binding to Both Poly(ADP-Ribose) and DNA by the Central BRCT Domain of XRCC1. Cell Rep. 2019 Jan;26(3):573-581.e5.

42. Abbotts R, Wilson DM 3rd. Coordination of DNA single strand break repair. Free Radic Biol Med. 2017 Jun;107(107):228-44.

43. Sukhanova MV, Khodyreva SN, Lebedeva NA, Prasad R, Wilson $\mathrm{SH}$, Lavrik OI. Human base excision repair enzymes apurinic/ apyrimidinic endonuclease1 (APE1), DNA polymerase beta and poly(ADP-ribose) polymerase 1: interplay between stranddisplacement DNA synthesis and proofreading exonuclease activity. Nucleic Acids Res. 2005 Feb;33(4):1222-9.

44. Dantzer F, de La Rubia G, Ménissier-De Murcia J, Hostomsky Z, de Murcia G, Schreiber V. Base excision repair is impaired in mammalian cells lacking Poly(ADP-ribose) polymerase-1. Biochemistry. 2000 Jun;39(25):7559-69.

45. Prasad R, Dyrkheeva N, Williams J, Wilson SH. Mammalian base excision repair: functional partnership between PARP-1 and APE1 in AP-site repair. PLoS One. 2015 May;10(5):e0124269.

46. Dutertre $M$, Lambert $S$, Carreira A, Amor-Guéret $M$, Vagner $S$. DNA damage: RNA-binding proteins protect from near and far. Trends Biochem Sci. 2014 Mar;39(3):141-9.

47. Yuan M, Eberhart CG, Kai M. RNA binding protein RBM14 promotes radio-resistance in glioblastoma by regulating DNA repair and cell differentiation. Oncotarget. 2014 May;5(9):2820-6.

48. Simon NE, Yuan M, Kai M. RNA-binding protein RBM14 regulates dissociation and association of non-homologous end joining proteins. Cell Cycle. 2017 Jun;16(12):1175-80.

49. Alfano L, Caporaso A, Altieri A, Dell'Aquila M, Landi C, Bini L, et al. Depletion of the RNA binding protein HNRNPD impairs homologous recombination by inhibiting DNA-end resection and inducing R-loop accumulation. Nucleic Acids Res. 2019 May;47(8):4068-85.

50. Taschuk F, Cherry S. DEAD-Box Helicases: Sensors, Regulators, and Effectors for Antiviral Defense. Viruses. 2020 Feb;12(2):181.

51. Sen ND, Gupta N, K Archer S, Preiss T, Lorsch JR, Hinnebusch AG. Functional interplay between DEAD-box RNA helicases Ded1 and Dbp1 in preinitiation complex attachment and scanning on structured mRNAs in vivo. Nucleic Acids Res. 2019 Sep;47(16):8785-806.

52. Song C, Hotz-Wagenblatt A, Voit R, Grummt I. SIRT7 and the DEAD-box helicase DDX21 cooperate to resolve genomic R loops and safeguard genome stability. Genes Dev. 2017 Jul;31(13):1370-81.

53. Li L, Germain DR, Poon HY, Hildebrandt MR, Monckton EA, McDonald D, et al. DEAD Box 1 Facilitates Removal of RNA and Homologous Recombination at DNA Double-Strand Breaks. Mol Cell Biol. 2016 Oct;36(22):2794-810.

54. Nott TJ, Petsalaki E, Farber P, Jervis D, Fussner E, Plochowietz A, et al. Phase transition of a disordered nuage protein generates environmentally responsive membraneless organelles. Mol Cell. 2015 Mar;57(5):936-47.

55. Hondele M, Sachdev R, Heinrich S, Wang J, Vallotton P, Fontoura $B M$, et al. DEAD-box ATPases are global regulators of phaseseparated organelles. Nature. 2019 Sep;573(7772):144-8.

56. Francia S, Michelini F, Saxena A, Tang D, de Hoon M, Anelli V, et al. Site-specific DICER and DROSHA RNA products control the DNA-damage response. Nature. 2012 Aug;488(7410):231-5.

57. McNeill DR, Whitaker AM, Stark WJ, Illuzzi JL, McKinnon $P J$, Freudenthal BD, et al. Functions of the major abasic endonuclease (APE1) in cell viability and genotoxin resistance. Mutagenesis. 2020 Feb;35(1):27-38.

58. Antoniali G, Malfatti MC, Tell G. Unveiling the non-repair face of the Base Excision Repair pathway in RNA processing: A missing link between DNA repair and gene expression? DNA Repair (Amst). 2017 Aug;56(June):65-74.

59. Vascotto C, Fantini D, Romanello M, Cesaratto L, Deganuto M, Leonardi A, et al. APE1/Ref-1 interacts with NPM1 within nucleoli and plays a role in the rRNA quality control process. Mol Cell Biol. 2009 Apr;29(7):1834-54.

60. Tell G, Wilson DM 3rd, Lee CH. Intrusion of a DNA repair protein in the RNome world: is this the beginning of a new era? Mol Cell Biol. 2010 Jan;30(2):366-71. 
61. Poletto M, Vascotto C, Scognamiglio PL, Lirussi L, Marasco D, Tell G. Role of the unstructured N-terminal domain of the hAPE1 (human apurinic/apyrimidinic endonuclease 1) in the modulation of its interaction with nucleic acids and NPM1 (nucleophosmin). Biochem J. 2013 Jun;452(3):545-57.

62. Lirussi L, Antoniali G, Vascotto C, D’Ambrosio C, Poletto M, Romanello M, et al. Nucleolar accumulation of APE1 depends on charged lysine residues that undergo acetylation upon genotoxic stress and modulate its BER activity in cells. Mol Biol Cell. 2012 Oct;23(20):4079-96.

63. Moor NA, Vasil'eva IA, Kuznetsov NA, Lavrik OI. Human apurinic/ apyrimidinic endonuclease 1 is modified in vitro by poly(ADPribose) polymerase 1 under control of the structure of damaged DNA. Biochimie. 2020 Jan;168:144-55.

64. López DJ, Rodríguez JA, Bañuelos S. Nucleophosmin, a multifunctional nucleolar organizer with a role in DNA repair. Biochim Biophys Acta Proteins Proteomics. 2020 Dec;1868(12):140532.

65. Ayyildiz D, Antoniali G, D’Ambrosio C, Mangiapane G, Dalla E, Scaloni A, et al. Architecture of The Human Ape1 Interactome Defines Novel Cancers Signatures. Sci Rep. 2020 Jan;10(1):28.

66. Abbasi S, Schild-Poulter C. Mapping the Ku Interactome Using Proximity-Dependent Biotin Identification in Human Cells. J Proteome Res. 2019 Mar;18(3):1064-77.

67. Arifuzzaman M, Maeda M, Itoh A, Nishikata K, Takita C, Saito $R$, et al. Large-scale identification of protein-protein interaction of Escherichia coli K-12. Genome Res. 2006 May;16(5):686-91.

68. Shchepachev V, Bresson S, Spanos C, Petfalski E, Fischer L, Rappsilber J, et al. Defining the RNA interactome by total RNA-associated protein purification. Mol Syst Biol. 2019 Apr;15(4):e8689.

69. Simabuco FM, Pavan IC, Pestana NF, Carvalho PC, Basei FL, Campos Granato D, et al. Interactome analysis of the human Cap-specific mRNA (nucleoside-2'-0-)-methyltransferase 1 (hMTr1) protein. J Cell Biochem. 2019 Apr;120(4):5597-611.

70. Li Y, Merkel CD, Zeng X, Heier JA, Cantrell PS, Sun M, et al. The $\mathrm{N}$-cadherin interactome in primary cardiomyocytes as defined using quantitative proximity proteomics. J Cell Sci. 2019 Feb;132(3):1-16.

71. Antoniali G, Serra F, Lirussi L, Tanaka M, D’Ambrosio C, Zhang $\mathrm{S}$, et al. Mammalian APE1 controls miRNA processing and its interactome is linked to cancer RNA metabolism. Nat Commun. 2017 Oct;8(1):797.

72. Poletto M, Lirussi L, Wilson DM 3rd, Tell G. Nucleophosmin modulates stability, activity, and nucleolar accumulation of base excision repair proteins. Mol Biol Cell. 2014 May;25(10):164152.

73. Tell G, Fantini D, Quadrifoglio F. Understanding different functions of mammalian AP endonuclease (APE1) as a promising tool for cancer treatment. Cell Mol Life Sci. 2010 Nov;67(21):3589-608.

74. Kristensen AR, Gsponer J, Foster LJ. A high-throughput approach for measuring temporal changes in the interactome. Nat Methods. 2012 Sep;9(9):907-9.

75. Piovesan D, Tabaro F, Paladin L, Necci M, Mičetić I, Camilloni C, et al. MobiDB 3.0: more annotations for intrinsic disorder, conformational diversity and interactions in proteins. Nucleic Acids Res. 2018 Jan;46 D1:D471-6.
76. Mészáros B, Erdős G, Szabó B, Schád É, Tantos Á, Abukhairan R, et al. PhaSePro: the database of proteins driving liquid-liquid phase separation. Nucleic Acids Res. 2020 Jan;48 D1:D360-7.

77. You K, Huang Q, Yu C, Shen B, Sevilla C, Shi M, et al. PhaSepDB: a database of liquid-liquid phase separation related proteins. Nucleic Acids Res. 2020 Jan;48 D1:D354-9.

78. Subramanian A, Tamayo P, Mootha VK, Mukherjee S, Ebert BL, Gillette MA, et al. Gene set enrichment analysis: a knowledgebased approach for interpreting genome-wide expression profiles. Proc Natl Acad Sci USA. 2005 Oct;102(43):15545-50.

79. Bindea G, Mlecnik B, Hackl H, Charoentong P, Tosolini M, Kirilovsky A, et al. ClueGO: a Cytoscape plug-in to decipher functionally grouped gene ontology and pathway annotation networks. Bioinformatics. 2009 Apr;25(8):1091-3.

80. Shannon P, Markiel A, Ozier O, Baliga NS, Wang JT, Ramage D, et al. Cytoscape: a software environment for integrated models of biomolecular interaction networks. Genome Res. 2003 Nov;13(11):2498-504.

81. Wegmann S, Eftekharzadeh B, Tepper K, Zoltowska KM, Bennett $\mathrm{RE}$, Dujardin S, et al. Tau protein liquid-liquid phase separation can initiate tau aggregation. EMBO J. 2018 Apr;37(7):1-21.

82. Aguzzi A, Altmeyer M. Phase Separation: Linking Cellular Compartmentalization to Disease. Trends Cell Biol. 2016 Jul;26(7):547-58.

83. Liberzon A, Subramanian A, Pinchback R, Thorvaldsdóttir H, Tamayo P, Mesirov JP. Molecular signatures database (MSigDB) 3.0. Bioinformatics. 2011 Jun;27(12):1739-40.

84. Liberzon A, Birger C, Thorvaldsdóttir H, Ghandi M, Mesirov JP, Tamayo P. The Molecular Signatures Database (MSigDB) hallmark gene set collection. Cell Syst. 2015 Dec;1(6):417-25.

85. Alberti S, Saha S, Woodruff JB, Franzmann TM, Wang J, Hyman AA. A User's Guide for Phase Separation Assays with Purified Proteins. J Mol Biol. 2018 Nov;430(23):4806-20.

86. Conicella AE, Zerze GH, Mittal J, Fawzi NL. ALS Mutations Disrupt Phase Separation Mediated by $\alpha$-Helical Structure in the TDP-43 Low-Complexity C-Terminal Domain. Structure. 2016 Sep;24(9):1537-49.

87. Jain A, Vale RD. RNA phase transitions in repeat expansion disorders. Nature. 2017 Jun;546(7657):243-7.

88. Elbaum-Garfinkle S. Matter over mind: liquid phase separation and neurodegeneration. J Biol Chem. 2019 May;294(18):7160-8.

89. Antoniali G, Lirussi L, Poletto M, Tell G. Emerging roles of the nucleolus in regulating the DNA damage response: the noncanonical DNA repair enzyme APE1/Ref- 1 as a paradigmatical example. Antioxid Redox Signal. 2014 Feb;20(4):621-39.

90. Whitaker AM, Stark WJ, Flynn TS, Freudenthal BD. Molecular and structural characterization of disease-associated APE1 polymorphisms. DNA Repair (Amst). 2020 Jul - Aug;9192:102867. 